\title{
Analysis of Electric Transport Mechanism of Barium Titanate by Impedance Spectroscopy
}

\author{
NUDRAT Humera ${ }^{a,}$ HADIA Noor ${ }^{b,},{ }^{,}$SAIRA Riaz ${ }^{c}$ and SHAHZAD Naseem ${ }^{d}$ \\ Centre of Excellence in Solid State Physics, University of Punjab, Lahore 54590, Pakistan \\ anudrathumera@gmail.com, bhadia.cssp@pu.edu.pk, 'saira.cssp@yahoo.com, \\ dshahzad_naseem@yahoo.com
}

\begin{abstract}
Keywords: Dielectric Properties, Impedance, Perovskite, Grains, Activation Energy, Hopping mechanism.
\end{abstract}

\begin{abstract}
Barium titanate (BTO) samples were prepared by nano milling based solid state reaction method. Dielectric behavior of BTO sample has been investigated by temperature dependent impedance spectroscopy. The impedance analysis of BTO samples confirms that hopping of electrons and defects coexisted in sample and contribution of grain and grain boundary to dielectric behavior is discussed. A single semicircular arc obtained by Nyquist plots whose radius decreased with increase in temperature illustrates the prominent role of grains. The activation energy of defects was calculated from Arrhenius plot. These defects may be due to oxygen vacancies and hopping between $\mathrm{Ti}^{+4}$ to $\mathrm{Ti}^{+3}$ ions.
\end{abstract}

\section{Introduction}

Dielectric ceramic is considered as significant material in the progress of microelectronic and microwave communication systems. In order to minimize the device size, it is necessary to have a material with high dielectric constant and a low-temperature coefficient of the permittivity. Thus, most research efforts have been moved towards perovskite structured materials because it has octahedral orientated cations such as $\mathrm{Ti}^{4+}, \mathrm{Nb}^{5+}$ and $\mathrm{Ta}^{5+}$ on the $\mathrm{B}$ sites. These cations contain three types of polarization mechanisms such as electronic, ionic, and dipolar which is reason of high values of dielectric constant. Its general formula is $\mathrm{ABO}_{3}$. Among these dielectric ceramics, Barium titanate (BTO) is an important functional material because of its piezoelectric and ferroelectric properties. It is widely used in various applications such as thermistors, dynamic random access memory (DRAM), actuators, sensors and multilayer ceramic capacitors (MLCC) [1-4]. Furthermore, the BTO has less toxic nature as compared to the other ceramic materials [5].

Various research groups worked on this material because it is appropriate for energy storage applications and their physical properties can be easily modified by controlling its particle size. In addition, particle size determines the electrical resistance of the material. It is interesting to study the change in dielectric properties by particle size. To control the particle size, nano-ball milling based solid-state is suitable method because it is simple, fast and cost effective method. In the ball milling, the grinding bowl, straddling on a turning support frame, also revolves about its axis. The forces produced by these rotations are applied to the milling balls and materials inside the bowl. Particle size decreased and produce microstrains at the same time in the material due to these forces.

We have previously reported structural and dielectric properties of BTO at room temperature with different milling frequencies [6]. To understand the role of grain and grain boundary in different mechanisms such as polarization, conductivity and relaxation process, BTO sample was prepared using optimized parameters of the previous work. In the present work, dielectric measurements were performed at different temperatures. The behavior of dielectric constant, tangent loss and AC conductivity are discussed at all temperatures and associated with different polarization and conduction mechanism. The contribution of grain is analyzed using impedance spectroscopy. The values of activation energies were calculated at different frequencies by means of Arrhenius plot and correlated with structural defects. 


\section{Experimental Details}

Barium titanate was prepared by solid state reaction. A mixture of $\mathrm{BaO}(99.99 \%$ pure, Sigma-Aldrich) and $\mathrm{TiO}_{2}(99.99 \%$, Merck) was prepared at room temperature.

$$
\mathrm{BaO}+\mathrm{TiO}_{2} \rightarrow \mathrm{BaTiO}_{3}
$$

The mixture was ball milled [Fritsch, Germany] with five rotating balls at $50 \mathrm{~Hz}$ milling frequency for two hours in a ceramic bowl. Then pellet was prepared a using hydraulic press by applying pressure $27.58 \mathrm{MPa}$ for 2 minutes. Area and thickness of pellet were $0.832 \mathrm{~cm}^{2}$ and $0.185 \mathrm{~cm}$, respectively. Afterwards, the samples were heated at $1200{ }^{\circ} \mathrm{C}$ in oxidizing environment for 24 hours. The Impedance analyzer (Wayne Kerr $6500 \mathrm{~B}$ ) was used to determine the frequency dependent dielectric parameters at different temperatures $\left(30^{\circ} \mathrm{C}\right.$ to $\left.210^{\circ} \mathrm{C}\right)$.

\section{Results and Discussion}

The Fig. 1 shows the temperature dependence of the dielectric constant at different frequencies. It is clear that the dielectric constant increases with increase in temperature. This increase in dielectric values can be attributed to the formation of insulating layer around the grains at high temperature. This layer is more resistive as compared to the grain [7]. The change in resistivity of grain (bulk) and layer (grain boundary) gives rise to the accumulation of charges which in turn results in the increase of internal polarization. Consequently, high dielectric value is observed at elevated temperatures. In addition, dielectric constant decreases as frequency is increased. Dielectric values decrease due to polarization. It can be explained as: there are four types (dipolar, electronic, ionic and interfacial) of polarization in dielectric materials. All the polarizations fully contribute to the applied electric field at low frequencies, whereas at high frequency response of different polarizations to the applied electric field becomes low leading to decrease in net polarization which reduces the dielectric values $[8,9]$. Temperature dependent tangent loss for BTO samples is shown in Fig. 2. Similar to dielectric constant, tangent loss also has large dispersion at low frequencies because the charge carriers require more energy to flow due to resistive behavior of grain boundaries which increases the energy losses in the low frequencies region.

The impedance analysis of BTO has been reported in the literature [10-12]. However, the difference lies in dielectric parameters from one report to the other often ascribed the different techniques. This variation is due to the effects of grain size, chemical inhomogeneity, grain and grain boundary impedance. Thus, complex impedance spectroscopy is suitable method to investigate the electrical behavior of ceramic materials and their temperature dependence. In order to analyze the electric properties, it is necessary to understand how the dielectric data transforms according to different formalism. The $\mathrm{Z}$ is suitable function to explore the resistive and/or conductive behavior of material. Fig. 3 shows the Nyquist plots of BTO at different temperatures. The data obtained at room temperature illustrates a straight line (not shown here) with large slope indicating the insulating behavior of BTO at room temperature. The slope reduced with the increase in temperature and semicircular arcs are formed by bending the slope towards the major $(Z)$ axis. As the temperature is increased, the radius of curvature decreased, which indicate the conducting nature at high temperature. However, the presence of a single semicircular arc indicates the grain interior (bulk) property of the material [13]. It is observed that the magnitude of $Z^{\prime}$ decreases with increase in temperature, which can be related to the increase in AC conductivity in the specimen. It is possible that defects such as oxygen vacancies result in the increase of AC conductivity. Thus, the role of oxygen vacancies is dominant at high temperature, whereas these defects are immobile at low temperature. The variation in the highest value of $Z$ " is due to the presence of temperature dependent relaxation process. 

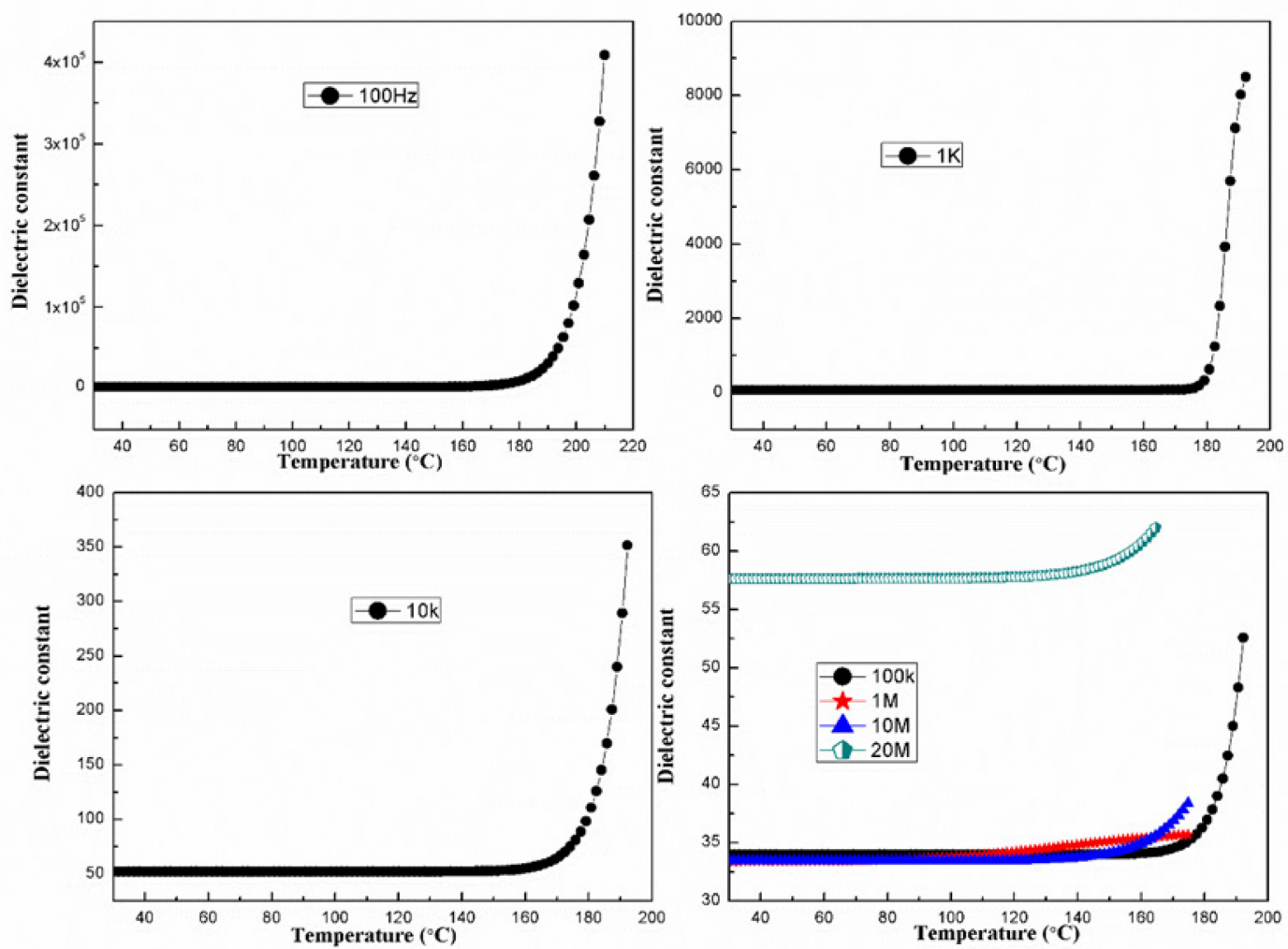

Fig. 1 Dielectric constant of BTO at various frequencies from $100 \mathrm{~Hz}$ to $20 \mathrm{MHz}$.
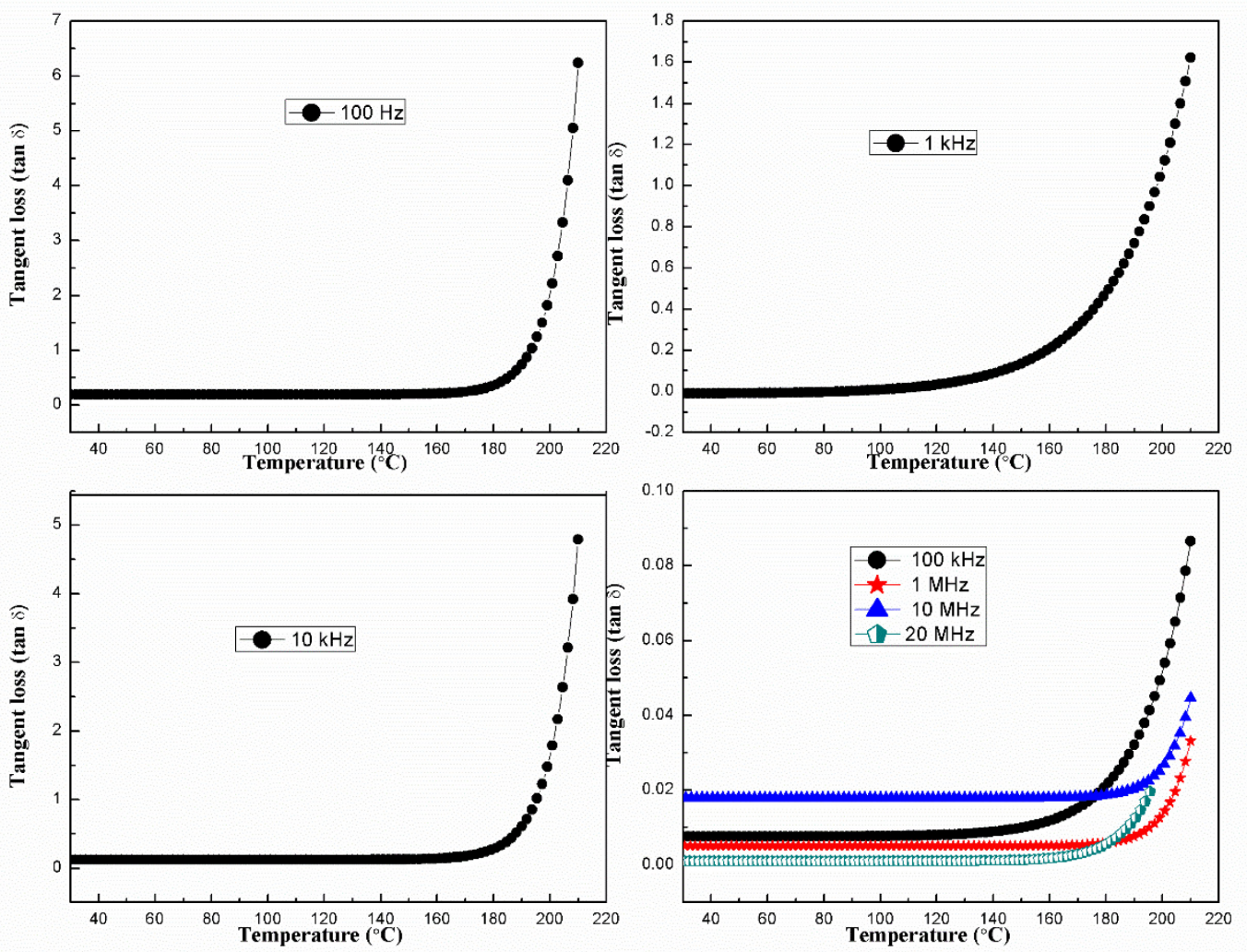

Fig. 2 Tangent loss of BTO at various frequencies from $100 \mathrm{~Hz}$ to $20 \mathrm{MHz}$. 


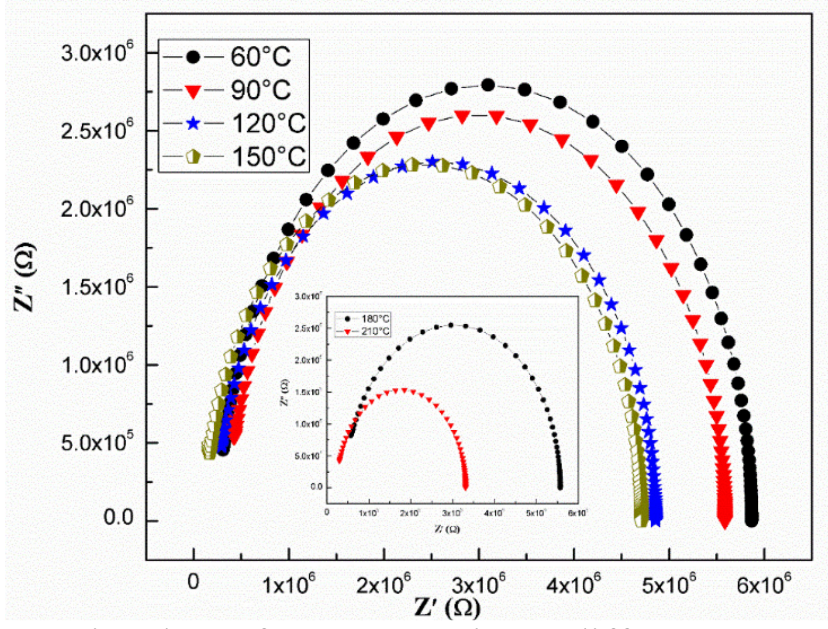

Fig. 3 Nyquist plots of BTO ceramics at different temperatures.

Fig. 4 demonstrates the variation of $\mathrm{AC}$ conductivity $\left(\sigma_{\mathrm{ac}}\right)$ with frequency at different temperatures from $30{ }^{\circ} \mathrm{C}$ to $210{ }^{\circ} \mathrm{C}$ which reveals that $\mathrm{AC}$ conductivity strongly depends on the frequency. The conductivity depends on the lattice defects in solids such as vacancies and dislocations as reported in previous studies $[14,15]$. There is a gradual increase in AC conductivity with increase in frequency of applied field due to the increase in hopping of electrons between ions. Furthermore, lattice defects can influence the motion of charges resulting in changed conductivity. The presence of $\mathrm{Ti}^{3+}$ ions and oxygen vacancies is responsible for hopping mechanism in BTO [16]. It is observed that the $\mathrm{AC}$ conductivity increased with increase in temperature. The increase in conductivity with temperature results in an increase in drift mobility of the charge carriers, which enhances the hopping mechanism [17]. The electrical conduction improvement at high temperature supports the impedance analysis results.

The plots of AC conductivity as a function of temperatures is shown in Fig. 5. It is clear from the figure that there are two regions which indicate the presence of different conduction mechanisms. Every region has its different activation energy at a particular frequency. The values of the activation energy of two regions at different frequencies were calculated according to the Arrhenius law [18].

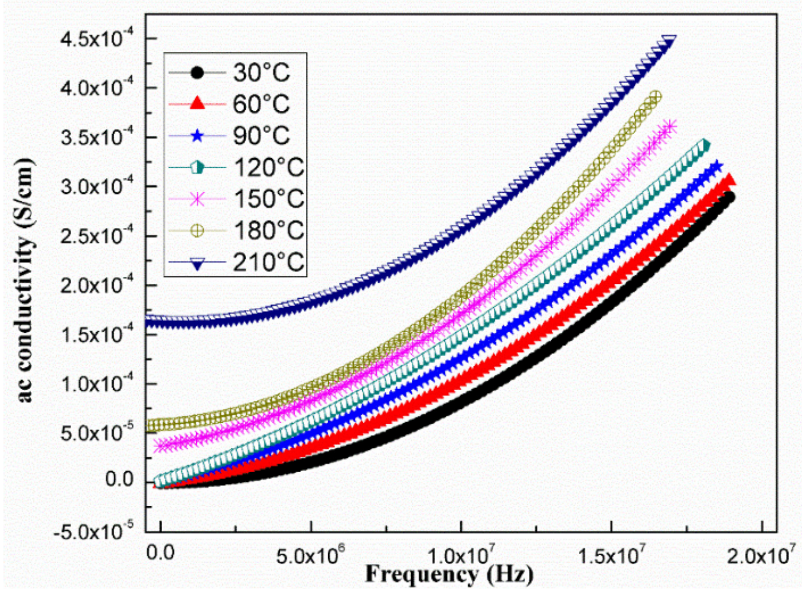

Fig. 4 Variation of AC conductivity vs. frequency at various temperatures.

$$
\sigma_{a c}=\sigma_{0} \exp \left(-\frac{E_{a}}{k_{B} T}\right)
$$

Where, $\sigma_{0}, \sigma_{\mathrm{ac}}, \mathrm{E}_{\mathrm{a}}, \mathrm{k}_{\mathrm{B}}$ and $\mathrm{T}$ are pre-exponential term, $\mathrm{AC}$ conductivity, activation energy, Boltzmann constant and temperature in Kelvin scale, respectively 


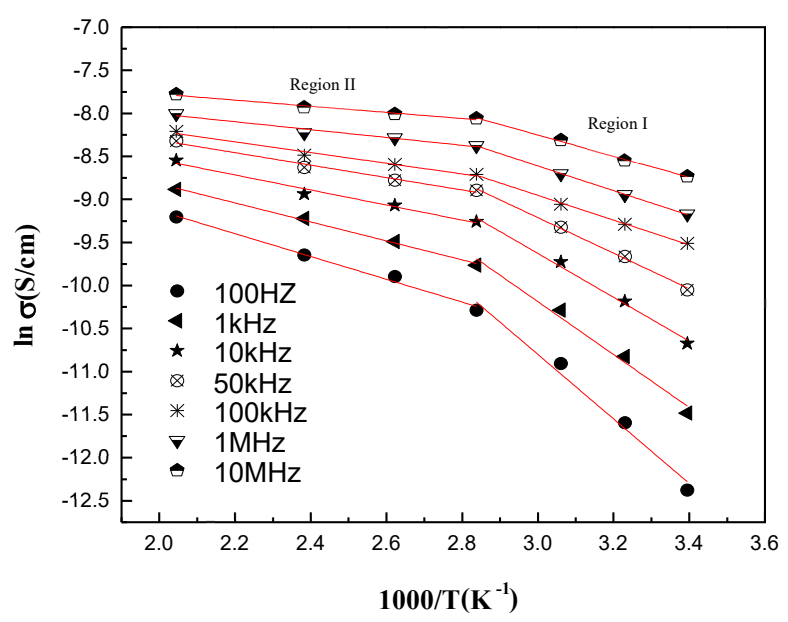

Fig. 5 Variation of AC conductivity vs. inverse of temperature at different frequencies for BTO. The calculated values of the activation energy at various frequencies are given in Table 1.

Table 1 Activation energy values $\left(\mathrm{E}_{\mathrm{a}}\right)$ for $\mathrm{AC}$ conduction calculated by Arrhenius plots for both regions indicated in Fig. 5 at various frequencies.

\begin{tabular}{c|c|c}
\hline $\mathbf{f}(\mathbf{H z})$ & $\mathbf{E}(\mathbf{m e V})$ of region I & $\mathbf{E}(\mathbf{m e V})$ of region II \\
\hline 100 & 323 & 114 \\
$1 \mathrm{k}$ & 265 & 95 \\
$10 \mathrm{k}$ & 219 & 75 \\
$50 \mathrm{k}$ & 178 & 62 \\
$100 \mathrm{k}$ & 124 & 54 \\
$1 \mathrm{M}$ & 124 & 39 \\
$10 \mathrm{M}$ & 106 & 31 \\
\hline
\end{tabular}

The values of activation energy at various frequencies increase in region-I as compared with region-II. The interaction between electron and/or hole-phonon enhances at low temperature due to the lattice deformation. This lattice deformation can be due to the presence of $\mathrm{Ti}^{3+}$ ions and oxygen vacancies. The induction of small polarons due to interaction between electron and/or hole-phonon is the reason for high value of activation energy. At high temperature, values of activation energy are found to vary from 31 to $114 \mathrm{meV}$. The activation energy in this region is associated with the hopping charge mechanism related with oxidation and reduction process. Furthermore, oxygen vacancies exhibit more mobility at high temperature and become activated to contribute in conduction process. The calculated values of energy are consistent with double ionized oxygen vacancies present in ceramic oxides described in literature for various ceramic oxides associated with hopping mechanism [19].

\section{Conclusions}

Barium titanate ceramic sample has been synthesized by nano-milling based solid state reaction technique. Frequency dependent dielectric measurements at different temperatures has been performed. Dielectric constant, tangent loss, AC conductivity and impedance analysis point out the relationship between temperature and microstructure effect which can be explained on the basis of Koop's theory. The contribution of grain (bulk) is observed in the material by Impedance spectroscopy using Nyquist plots. The mobility of the structural defects through hopping is the origin of electrical conduction in BTO. The activation energy values of these defects were calculated by Arrhenius plots. Highest activation energy value at low frequency indicated the dominant role of grains at high frequency. 


\section{References}

[1] J.B. Xu, J.W. Zhai, X. Yao, Structure and dielectric nonlinear characteristics of $\mathrm{BaTiO}_{3}$ thin films prepared by low temperature process, J. Alloys Compd., 467 (2009) 567-571.

[2] E.K. Nyutu, C.H. Chen, P.K. Dutta, S.L. Suib, Effect of microwave frequency on hydrothermal synthesis of nanocrystalline tetragonal barium titanate, J. Phys. Chem. C, 112 (2008) 9659-9667.

[3] H. Fan, L. Liu, Optimizating design of the microstructure of sol-gel derived $\mathrm{BaTiO}_{3}$ ceramics by artificial neural networks, J. Electroceram., 22 (2009) 291-296.

[4] F. Wan, J.G. Han, Z.Y. Zhu, Dielectric response in ferroelectric $\mathrm{BaTiO}_{3}$, Phys. Lett. A, 372 (2008) 2137-2140.

[5] A.K. Nath, C. Jiten, Influence of ball milling parameters on the particle size of barium titanate nanocrystalline powders, Physi. B, 405 (2010) 430-434.

[6] S. N. Humera, H. Noor, S. Riaz and S. Naseem, Dielectric and structural analysis of barium titanate nanoparticles prepared by nano ball milling technique, Presented at The 2016 World Congress on Adv.Civ. Envir. Mat. Res., August 28-September 1, 2016, ICC Jeju, Jeju Island, Korea.

[7] D.C. Sinclair, et al., $\mathrm{CaCu}_{3} \mathrm{Ti}_{4} \mathrm{O}_{12}$ : One-step internal barrier layer capacitor, Appl. Phys. Lett., 80 (2002) 2153-2155.

[8] J.C. Anderson, Dielectrics, Spottiswoode, Ballantyne \& Co Ltd, London and Colcheester, 1964.

[9] A.Verma, O.P. Thankur, C. Parkash, et al., Temperature dependence of electrical properties of nickel-zinc ferrites processed by the citrate precursor technique, Mater. Sci. Eng. B, 116 (2005) 16.

[10] S. Neogi, U. Chowdhury, A. Kumar Chakraborty, J. Ghosh, Effect of mechanical milling on the structural and dielectric properties of $\mathrm{BaTiO}_{3}$ powders, Micro \& Nano Lett., 10 (2015) 109-114.

[11] S.S. Kumbhar, M.A. Mahadik, et al., Structural and electrical properties of barium titanate $\left(\mathrm{BaTiO}_{3}\right)$ thin films obtained by spray pyrolysis method, Mater. Sci.-Poland., 33 (2015) 852-861.

[12] S. Kumar, N. Ahlawat, N. Ahlawat, Microwave sintering time optimization to boost structural and electrical properties in $\mathrm{BaTiO}_{3}$ ceramics, J. Integr. Sci. Technol., 4 (2016) 10-16.

[13] Y. D. Kolekar, L. J. Sanchez, C. V. Ramana, Dielectric relaxations and alternating current conductivity in manganese substituted cobalt ferrite, J. Appl. Phys., 115 (2014) 144106-11.

[14] N. R. Kalidindi, K. Kamala Bharathi, C. V. Ramana, The disordering effect of Ti observed in the microstructure and electrical properties of $\mathrm{W}_{0.95} \mathrm{Ti}_{0.05} \mathrm{O}_{3}$ thin films, Appl. Phys. Lett., 14 (2010) 142107.

[15] R. S. Vemuri, K.K. Bharathi, S.K. Gullapalli, C. V. Ramana, Effect of structure and size on the electrical properties of nanocrystalline $\mathrm{WO}_{3}$ films. ACS Appl. Mater. \& Interfac., 2 (2010) 2623-2628.

[16] S. B. Narangi, D. Kaur, K. Pubby, Effect of substitution of samarium and lanthanum on dielectric and electrical properties of barium titanate, Ferroelectrics., 486 (2015) 74-85.

[17] R. Nongjai, S. Khan, K. Asokan, H. Ahmed, I. Khan, Magnetic and electrical properties of In doped cobalt ferrite nanoparticles, J. Appl. Phys., 112 (2012) 084321-8.

[18] W. D. Kingery, Introduction to Ceramics, Wiley, New York, NY, USA, 1960.

[19] O. Raymond, R. Font, N. Suárez-Almodovar, J. Portelles, J.M. Siqueiros, Frequencytemperature response of ferroelectromagnetic $\mathrm{Pb}\left(\mathrm{Fe}_{1 / 2} \mathrm{Nb}_{1 / 2}\right) \mathrm{O}_{3}$ ceramics obtained by different precursors. Part I. Structural and thermo-electrical characterization. J. Appl. Phys., 97 (2005) 084107-8. 